\title{
Endurance training limits the functional alterations of heart rat mitochondria submitted to in vitro anoxia-reoxygenation
}

\author{
António Ascensão ${ }^{\text {a,* }}$, José Magalhães ${ }^{\text {a }}$, José M.C. Soares ${ }^{\text {a }}$, Rita Ferreira ${ }^{\text {a }}$, Maria J. Neuparth ${ }^{\text {a }}$, \\ Franklim Marques ${ }^{\mathrm{c}, \mathrm{d}}$, Paulo J. Oliveira ${ }^{\mathrm{e}, \mathrm{f}}$, José A. Duarte ${ }^{\mathrm{a}, \mathrm{b}}$ \\ ${ }^{a}$ Department of Sport Biology, Faculty of Sport Sciences, University of Porto, Rua Dr. Plácido Costa, 91, 4200-450 Porto, Portugal \\ ${ }^{\mathrm{b}}$ Center for Research in Physical Activity and Leisure, Faculty of Sport Sciences, University of Porto, Portugal \\ ${ }^{\mathrm{c}}$ Department of Biochemistry and Clinical Analysis, Faculty of Pharmacy, University of Porto, Portugal \\ ${ }^{\mathrm{d}}$ Institute for Molecular and Cell Biology, University of Porto, Portugal \\ ${ }^{\mathrm{e}}$ Department of Zoology, University of Coimbra, Portugal \\ ${ }^{\mathrm{f}}$ Centre of Neurosciences and Cell Biology, University of Coimbra, Portugal
}

Received 17 January 2005; received in revised form 8 April 2005; accepted 4 June 2005

Available online 14 July 2005

\begin{abstract}
Background: Studies analysing the effect of endurance training on heart mitochondrial function submitted to in vitro anoxia-reoxygenation (A-R) are missing. The present study aimed to investigate the effect of moderate endurance treadmill training (14 weeks) against rat heart mitochondrial dysfunction induced by in vitro A-R.

Methods: Respiratory parameters (state 3, state 4, ADP/O and respiratory control ratio- $\mathrm{RCR}$ ) and oxidative damage markers (carbonyl groups and malondialdehyde) were determined in isolated mitochondria before and after 1 min anoxia followed by 4 min reoxygenation. Levels of heat shock protein $60 \mathrm{kDa}$ (HSP60) and $70 \mathrm{kDa}$ (HSP70) were measured before A-R in mitochondria and whole muscle homogenate, respectively.

Results: A-R significantly impaired the rate of state 3 and state 4 respiration, as well as the RCR and ADP/O in the sedentary group. However, mitochondrial state 3 respiration was significantly higher in trained than in the sedentary group both before and after A-R. The impairments in RCR, ADP/O ratio and state 4 induced by A-R in sedentary group were significantly attenuated in endurance-trained group. The inhibition of state 4 induced by GDP was significantly higher in trained than in sedentary group. Oxidative modifications of mitochondrial proteins and phospholipids were found in sedentary group after A-R, although limited in trained group. Increased levels of mitochondrial HSP60 and tissue HSP70 accompanied the lower decrease in the respiratory function after A-R observed in trained group. Conclusion: We therefore concluded that endurance training limited the impairments on rat heart mitochondria caused by the oxidant insult inflicted by in vitro A-R.
\end{abstract}

(C) 2005 Elsevier Ireland Ltd. All rights reserved.

Keywords: Cardiac mitochondria; Exercise; Respiration; Oxidative phosphorylation; Oxidative damage; Heat shock proteins

\section{Introduction}

It is well known that severe stimuli to the heart can cause structural and functional cardiac muscle impairments [1] and that, among other cell compartments, mitochondria are

\footnotetext{
* Corresponding author. Tel.: +351 225074774; fax: +351 225500689 .

E-mail address: aascensao@fcdef.up.pt (A. Ascensão).
}

involved in the mechanisms related to some of these heart dysfunctions [2,3]. Actually, mitochondria are the cells' powerhouses where the energy required to drive the endergonic and vital biochemical processes of cell life is produced through a well-coupled mechanism of oxidative phosphorylation [4]. Coupled with ATP synthesis, mitochondria are also critical organelles involved in the modulation of osmotic regulation, cell redox status and $\mathrm{pH}$ control, signal transduction, and in the establishment of 
$\mathrm{Ca}^{2+}$ homeostasis with consequent implications in the cell fate [5].

It is often stated that regular exercise could constitute an excellent tool in the prevention of several heart dysfunctions as it increases the tolerance of cardiac tissue against stress conditions [6,7]. Since exercise represents an important heart mitochondrial stress-induced stimuli, it is possible that the structural and biochemical adaptations induced by training in these organelles, such as increased volume density, heat shock protein (HSP) expression and the upregulation of antioxidant enzymes activity, could be related to the training-induced cardiac cross tolerance effect [810]. Therefore, it could be expected that these biochemical and structural adaptations parallel with improvements in several features of mitochondria functionality, including the respiratory function [11]. However, the influence of training on heart mitochondrial respiration has been scarcely addressed, namely alterations of basic parameters like state 4 and state 3 respiration, $\mathrm{ADP} / \mathrm{O}$ and in the respiratory control ratio (RCR). Besides the study conducted by Leichtweis et al. [12] reporting enhanced mitochondrial respiratory susceptibility after an atypical daily vigorous swimming training ( $6 \mathrm{~h} /$ day, 5 days/week ${ }^{1}, 8-9$ weeks), the limited number of studies using moderate training workloads did not find any positive changes in the ratio between uncoupled and basal respiration [13]. Furthermore, it is also possible that the standard respiratory assays evaluated in isolated energized mitochondria are not sufficiently accurate to detect hypothetical improvements in respiratory function induced by chronic training in cardiac mitochondria and thus, an additional in vitro stimulus may be necessary to highlight possible differences in mitochondrial functional response.

In vitro anoxia-reoxygenation (A-R) of isolated mitochondria is a standard and well-established deleterious stimulus, mediated by oxidative stress $[14,15]$ that allows the exclusion of other cytosolic defense systems. Together with ADP-induced stimulation of mitochondria oxidative phosphorylation, constitutes a supplementary stress stimulus. Accordingly, it is possible that this model will be more sensitive to analyse the role of endurance training on respiratory rates, since in these conditions heart mitochondria from both sedentary and trained animals are submitted to an extra in vitro stimulus. Because the release of reactive oxygen species (ROS) is central to the mechanism of A-R-induced mitochondrial damage [14,15], it is logical to consider training-induced improved cardiac cell and mitochondria defences $[6,16,17]$ as one of the possible candidates to counteract A-R-induced respiratory impairments. Therefore, the purpose of the present study was to analyse the effect of moderate endurance treadmill training on the heart mitochondrial respiratory susceptibility to in vitro A-R and relate the findings with the levels of mitochondrial oxidative damage. Moreover, since the gene transfection and hyperthermic stress-mediated overexpression of cardiac HSP60 and HSP70 have been extensively reported to result in enhanced myocardial mitochondrial tolerance [18], we also investigated whether traininginduced HSP overexpression would correspond to mitochondrial respiratory protection.

\section{Methods}

\subsection{Sample}

Twenty Wistar male rats (aged 6-8 weeks, weighting $200 \mathrm{~g}$ at the beginning of the experiments) were used. During the experimental protocol, the animals were housed in collective cages ( 2 rats per cage) and were maintained in a room at normal atmosphere $\left(21-22{ }^{\circ} \mathrm{C} ; \sim 50-60 \%\right.$ humidity) receiving food and water ad libitum in $12 \mathrm{~h}$ light/dark cycles. The animals were randomly divided in two groups: Trained (T) and sedentary (S). Only male animals were used because of female estrogen protective effect on cardiac tissue, as reported elsewhere [19]. The Ethics Committee of the Scientific Board of Faculty of Sport Sciences, University of Porto, Portugal, approved the experimental protocol, which followed the Guidelines for Care and Use of Laboratory Animals in research.

\subsection{Endurance training protocol}

The animals from $\mathrm{T}$ group were exercised 5 days a week (Monday to Friday) for 14 weeks on a motor driven treadmill. Both the treadmill speed and grade were gradually increased over the course of the 14-week training period (Table 1). The protocol included 5 days of habituation to the treadmill with $10 \mathrm{~min}$ of running at $25 \mathrm{~m} / \mathrm{min}$ and $0 \%$ grade, with daily increases of 5-10 min until $30 \mathrm{~min}$ was achieved. Habituation was followed by one consecutive week of continuous running ( $30 \mathrm{~min} /$ day) at $25 \mathrm{~m} / \mathrm{min}$ and $0 \%$ grade and was gradually increased until $60 \mathrm{~min} /$ day on the second week. This protocol proved to be efficient in rising cardiac

Table 1

Exercise training protocol

\begin{tabular}{|c|c|c|c|c|c|c|c|c|c|c|c|c|c|c|}
\hline & \multicolumn{14}{|c|}{ Weeks of training } \\
\hline & 1 & 2 & 3 & 4 & 5 & 6 & 7 & 8 & 9 & 10 & 11 & 12 & 13 & 14 \\
\hline Exercise duration (min/day) & 30 & 60 & 90 & 90 & 90 & 90 & 90 & 90 & 90 & 90 & 90 & 90 & 90 & 90 \\
\hline Treadmill speed $(\mathrm{m} / \mathrm{min})$ & 25 & 25 & 30 & 30 & 30 & 30 & 30 & 30 & 30 & 30 & 30 & 30 & 30 & 30 \\
\hline$\%$ Grade (treadmill inclination) & 0 & 0 & 0 & 3 & 6 & 6 & 6 & 6 & 6 & 6 & 6 & 6 & 6 & 6 \\
\hline
\end{tabular}

Exercise-trained animals were exercised 5 days/week on a motorized treadmill for 14 weeks. 
dimensions (hypertrophy) and in antioxidant biochemical alterations protecting cardiac tissue during in vivo I/R [10]. The animals from $\mathrm{S}$ group were not exercised but were placed on a non-moving treadmill five times per week (10$30 \mathrm{~min} / \mathrm{session}$ ) with the purpose of homogenizing the possible environment stress induced by treadmill without promoting any physical training adaptations.

\subsection{Animals sacrifice heart and soleus extractions}

Twenty-four hours after the last exercise bout, the animals were sacrificed by cervical dislocation. After a quick opening chest, rat hearts were then rapidly excised, rinsed, carefully dried and weighted. A portion of approximately $20-25 \mathrm{mg}$ of cardiac ventricles and one soleus muscle were separated, homogenized in homogenization buffer $(0.05 \mathrm{M}$ Tris, $0.03 \mathrm{M}$ L-serine, $0.06 \mathrm{M}$ boric acid, tissue/buffer ratio of $100 \mathrm{mg} / \mathrm{mL}, \mathrm{pH}$ 7.6) using a Teflon pestle on a motor-driven Potter-Elvehjem glass homogenizer at $0-4{ }^{\circ} \mathrm{C}$ at three to five times for $5 \mathrm{~s}$ at speed low setting, with a final burst at a higher speed setting. Both homogenates were centrifuged $\left(2 \mathrm{~min}\right.$ at $2000 \times g, 4^{\circ} \mathrm{C}$, in order to eliminate cellular debris) and the resulting supernatant was stored at $-80{ }^{\circ} \mathrm{C}$ for later determination of HSP70 expression (cardiac ventricle) and citrate synthase activity (soleus). Protein content from cardiac muscle and soleus homogenates were assayed using bovine serum albumin as standard according to Lowry et al. [20].

\subsection{Isolation of rat heart mitochondria}

From the remaining heart ventricles, rat heart mitochondria of each group (one from sedentary and one from trained heart) were daily prepared in parallel using conventional methods of differential centrifugation [21] as follows. Briefly, the animals were sacrificed as above stated and the heart was immediately excised and finely minced in an ice-cold isolation medium containing $250 \mathrm{mM}$ sucrose, 0.5 $\mathrm{mM}$ EGTA, $10 \mathrm{mM}$ Hepes-KOH $(\mathrm{pH}$ 7.4) and $0.1 \%$ defatted BSA (Sigma no. A-7030). The minced blood free tissue was then resuspended in $40 \mathrm{~mL}$ of isolation medium containing $1 \mathrm{mg}$ protease subtilopeptidase A Type III (Sigma P-5380) per gram of tissue and homogenized with a tightly fitted homogenizer (Teflon: glass pestle). The suspension was incubated for $1 \min \left(4^{\circ} \mathrm{C}\right)$ and then rehomogenized. The homogenate was then centrifuged at $14,500 \times g$ for $10 \mathrm{~min}$. The supernatant fluid was decanted and the pellet, essentially devoided of protease, was gently re-suspended in its original volume $(40 \mathrm{~mL})$ with a loosefitting homogenizer. The suspension was centrifuged at $750 \times g$ for $10 \mathrm{~min}$. and the resulting supernatant was centrifuged at $12,000 \times g$ for $10 \mathrm{~min}$. The pellet was resuspended using a paintbrush and re-pellet at $12,000 \times g$ for 10 min. EGTA and defatted BSA were omitted from the final washing medium. Mitochondrial protein content was determined by the Biuret method calibrated with BSA. The isolation procedures were performed within approximately 1 $\mathrm{h}$ at $0-4{ }^{\circ} \mathrm{C}$. Aliquots of mitochondrial suspension were separated and frozen at $-80{ }^{\circ} \mathrm{C}$ for later determination of basal carbonyls, malondialdehyde (MDA) and HSP60 contents (see Fig. 1A).

The remaining mitochondrial suspensions were used within $4 \mathrm{~h}$ and were maintained on ice $\left(0-4{ }^{\circ} \mathrm{C}\right)$ throughout this period. Isolation procedures yielded well-coupled mitochondria: RCR of isolated mitochondria varied from 7-10 (with glutamate-malate) or 3-4 (with succinate plus rotenone) for controls, as determined according to the method of Estabrook [22]. These mitochondrial suspensions were used to analyse the influence of uncoupling proteins (UCP) on basal mitochondrial respiration and for testing the influence of in vitro A-R in each group (see Fig. 1A).

\subsection{Mitochondrial oxygen consumption assays}

Mitochondrial respiratory function was measured polarographically, at $25{ }^{\circ} \mathrm{C}$, using a Clark-type oxygen electrode (Hansatech DW 1, Norfolk, UK). Reactions were conducted in $0.75 \mathrm{~mL}$ closed thermostatic and magnetically stirred glass chamber containing $0.5 \mathrm{mg}$ of mitochondrial protein in a respiration buffer containing $65 \mathrm{mM} \mathrm{KCl}, 125 \mathrm{mM}$ sucrose, $10 \mathrm{mM}$ Tris, $20 \mu \mathrm{M}$ EGTA, $2.5 \mathrm{mM} \mathrm{KH}_{2} \mathrm{PO}_{4}$, pH 7.4.After 1-min equilibration period, mitochondrial respiration was initiated by adding glutamate and malate to a final concentration of 10 and $5 \mathrm{mM}$ each, respectively. We measured some of the standard respiratory parameters that include state 3 respiration (oxygen consumption in the presence of externally added $444 \mu \mathrm{M}$ ADP); state 4 (respiration rate observed upon complete ADP phosphorylation to ATP); RCR (a measure of the dependence of the respiratory rate on ATP synthesis and calculated as the ratio between the rate of oxygen consumption during state 3 and state 4) and ADP/O (a measure of mitochondrial oxidative phosphorylation efficiency that is defined as the ratio between the ADP added and oxygen consumed during ADP phosphorylation). The RCR and the ADP/O ratios were calculated according to Estabrook [22], using $474 \mathrm{ng}$ atom $\mathrm{O} / \mathrm{ml}$ as the value of solubility of oxygen at $25^{\circ} \mathrm{C}$ in doubly distilled water.

\subsection{Anoxia and reoxygenation}

Anoxia was performed by mitochondria $(0.5 \mathrm{mg} / \mathrm{mL})$ consuming the oxygen content in the reaction medium after two ADP pulses into a close incubation chamber at $25^{\circ} \mathrm{C}$. Glutamate $(10 \mathrm{mM})$ and malate $(5 \mathrm{mM})$ were used as substrates. Energized cardiac mitochondria were stimulated with the first $444 \mu \mathrm{M}$ ADP pulse to obtain pre-anoxia respiratory rates. The anaerobic conditions were reached in state 4 through the addition of a second ADP pulse $(1 \mathrm{mM})$ and the period of anoxia was set to 1 min (Fig. 1). Anoxia was followed by $4 \mathrm{~min}$ of in vitro reoxygenation by exposing the stirred medium containing the previously 


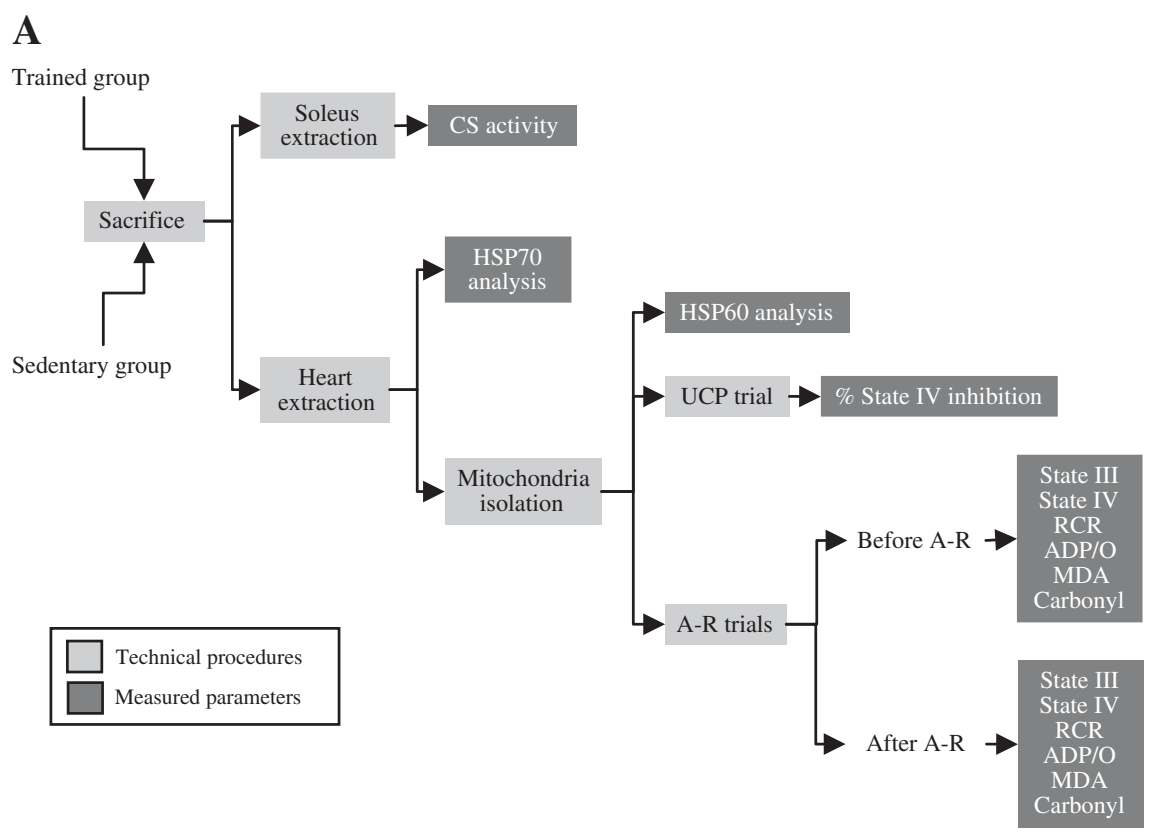

B

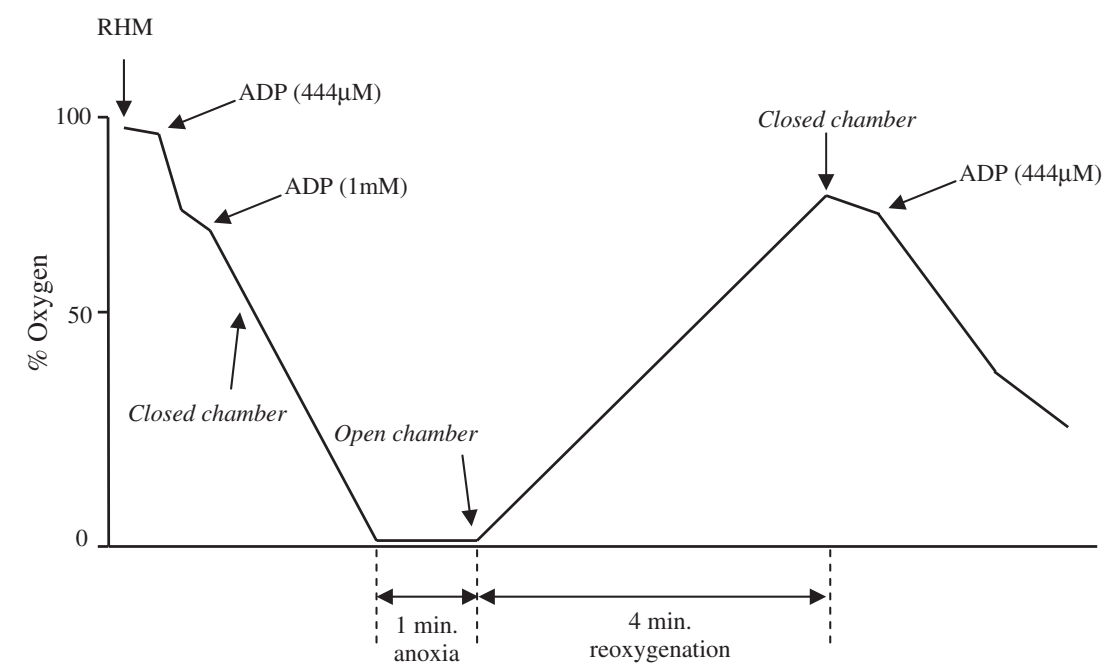

Fig. 1. Experimental protocol. (A) Sequence of the technical procedures and the 10 independent measured parameters from each group or condition. (B) Typical polarographic oxygen electrode traces obtained in the A-R model. Oxygen consumption rates of energized (10 mM glutamate $+5 \mathrm{mM}$ malate) rat heart mitochondria (RHM) isolated from trained and sedentary rats were determined at $25{ }^{\circ} \mathrm{C}$ before and after stimulation through A-R. State 3 and state 4 respiration, respiratory control ratio (RCR) and $\mathrm{ADP} / \mathrm{O}$ were calculated in both pre-anoxia (after the addition of the 1st ADP pulse) and in post-reoxygenation conditions (after another ADP pulse).

energized mitochondria to air (open chamber). Then, the polarographic oxygraph chamber was closed and the respiratory activities were measured again after the addition of another $444 \mu \mathrm{M}$ ADP pulse as shown in Fig. 1.

To analyse the susceptibility of heart mitochondria to A$\mathrm{R}$-mediated oxidative damage, mitochondria aliquots were separated and frozen at $-80{ }^{\circ} \mathrm{C}$ for later determination of post A-R carbonyls and MDA contents (see Fig. 1A).

With the purpose of ensuring whether the found changes were influenced by the in vitro mitochondria aging during respiratory assay, all the above referred respiratory parameters were also measured, in an independent trial (time- control trial), after 15-20 min incubation period in a stirred medium containing previously energized mitochondria exposed to air (Fig. 2). This time control was achieved in the presence of two pulses of ADP in order to mimic the pre-anoxic conditions reported in Fig. 1B.

\subsection{UCP-mediated basal respiration}

In an independent trial performed with glutamate (10 $\mathrm{mM})$, malate $(5 \mathrm{mM})$ and ADP $(444 \mu \mathrm{M})$, state 4 respiration was determined and cardiac mitochondria were subsequently exposed to the addition of GDP $(1 \mathrm{mM})$ to inhibit 


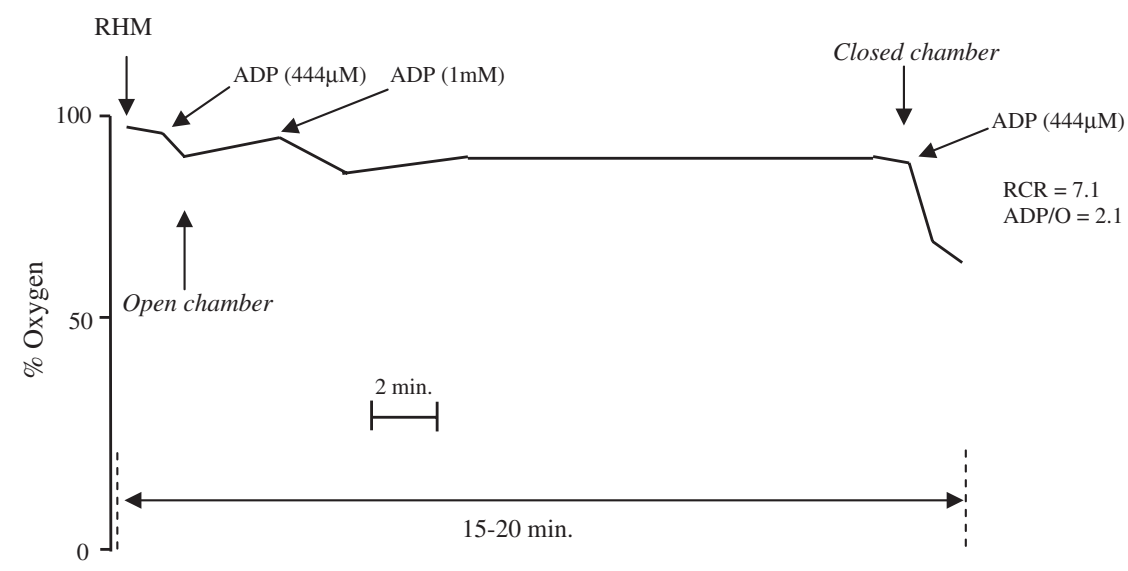

Fig. 2. Typical polarographic oxygen electrode traces obtained in time control trials. Respiratory rates corresponding to oxygen consumption of energized (10 $\mathrm{mM}$ glutamate $+5 \mathrm{mM}$ malate) rat heart mitochondria (RHM) isolated from trained and sedentary rats were determined at $25^{\circ} \mathrm{C}$ after $15-20$ min of incubation in a stirred reaction medium exposed to air. State 3 and state 4 respiration, respiratory control ratio (RCR) and ADP/O were calculated after the end of incubation period.

uncoupling proteins (UCP). The addition of this inhibitor allowed testing the relative contribution of these membrane proteins to proton leak through the mitochondrial inner membrane, which may contribute to state 4 respiration, as well to a possible membrane-linked system related to the induction of the so-called mild uncoupling [23].

\subsection{Lipid peroxidation evaluation}

Lipid peroxidation was measured by determining the levels of lipid peroxides as the amount of thiobarbituric acid reactive substances (TBARS) formed according to Rohn et al. [24], with some modifications. Mitochondrial protein (0.5 $\mathrm{mg}$ ) was incubated, at $25{ }^{\circ} \mathrm{C}$, in $500 \mu \mathrm{l}$ of a medium consisting of $175 \mathrm{mM} \mathrm{KCl}, 10 \mathrm{mM}$ Tris, $\mathrm{pH}$ 7.4. Samples of $50 \mu \mathrm{l}$ were taken and mixed with $450 \mu \mathrm{l}$ of a TBARS reagent (1\% thiobarbituric acid, $0.6 \mathrm{~N} \mathrm{HCl}, 0.0056 \%$ butylated hydroxytoluene). The mixture was heated at $80-90{ }^{\circ} \mathrm{C}$ during $15 \mathrm{~min}$, and recooled in ice for $10 \mathrm{~min}$ before centrifugation in Eppendorf centrifuge $(1500 \times g, 5 \mathrm{~min})$. Lipid peroxidation was estimated by the appearance of TBARS spectrophotometrically quantified at $535 \mathrm{~nm}$. The amount of TBARS formed was calculated using a molar extinction coefficient of $1.56 \times 10^{5} \mathrm{M}^{-1} \mathrm{~cm}^{-1}$ and expressed as nanomoles of MDA per milligram of protein [25].

\subsection{Analysis of protein carbonylation, HSP60 and HSP70}

To determine the levels of HSP60 and HSP70 in the heart mitochondria and cardiac muscle, respectively, a certain volume of the suspension/homogenate correspondent to 10 $\mu \mathrm{g}$ protein was resolved by SDS-PAGE $(12.5 \%$ acrylamide gels of $1 \mathrm{~mm}$ thickness) as described by Laemmli [26] and electroblotted onto nitrocellulose membranes according to Locke et al. [27]. The immunoblotts were probed with $1: 2000$ dilution of monoclonal anti-HSP 60 (Calbiochem) and monoclonal anti-HSP70 (Clone BRM-22, Sigma) and with 1:500 dilution of the secondary antibody (anti-mouse IgG peroxidase conjugate, Sigma, St. Louis, USA).

For protein carbonyl derivatives assay, a certain cardiac mitochondrial suspension volume (V) containing $20 \mu \mathrm{g}$ of protein was derivatised with dinitrophenylhydrazine (DNPH). Briefly, the sample was mixed with 1 $\mathrm{V}$ of $12 \%$ SDS plus $2 \mathrm{~V}$ of $20 \mathrm{mM}$ DNPH $10 \%$ TFA, followed by $30 \mathrm{~min}$ of dark incubation, after which 1.5 $\mathrm{V}$ of $2 \mathrm{M}$ Tris $/ 18.3 \%$ of $\beta$-mercaptoethanol were added. A negative control was simultaneously prepared for each sample. After diluting the derivatised proteins in TBS to obtain a final concentration of $0.001 \mu \mathrm{g} / \mu \mathrm{L}$, a $100 \mathrm{~mL}$ volume was slot-blotted into a Hybond-PVDF membrane. Immunodetection of carbonyls was then performed using rabbit anti-DNP (DAKO) as the first antibody, and antirabbit IgG-Peroxidase (Amersham Pharmacia) as the second antibody.

The bands were visualized by treating the immunoblotts with ECL chemiluminescence reagents (Amersham, Pharmacia Biotech, Buckinghamshine, UK), according to the supplier's instructions, followed by exposure to X-ray films (Sigma, Kodak Biomax Light Film, St. Louis, USA). The films were analyzed with QuantityOne Software (Bio Rad).

Table 2

Effects of endurance running training on rat body and heart weights as well as on the activity of skeletal muscle citrate synthase

\begin{tabular}{|c|c|c|c|c|}
\hline Groups & Body weight $(\mathrm{g})$ & Heart weight $(\mathrm{g})$ & Heart/body ratio $\left(\times 10^{-4}\right)$ & 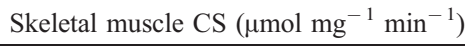 \\
\hline Sedentary & $514.66 \pm 16.3$ & $0.96 \pm 0.021$ & $18.8 \pm 0.44$ & $0.017 \pm 0.001$ \\
\hline Trained & $425.0 \pm 6.45 *$ & $1.29 \pm 0.054 *$ & $30.4 \pm 0.10 *$ & $0.056 \pm 0.004 *$ \\
\hline
\end{tabular}

Data represent the means \pm SEM for body and cardiac weights as well as for skeletal muscle citrate synthase (CS) activity.

$* p<0.05$ trained vs. sedentary. 
Optical density results were expressed as percentage variation of control values (sedentary group).

\subsection{Citrate synthase activity}

Soleus citrate synthase activity was measured using the method proposed by Coore et al. [28] by spectrophotometrically (412 nm) measuring the amount of 5,5-dithiobis (2-nitrobenzoate) that reacted with acetyl-CoA upon release from the reaction of acetyl-CoA with oxaloacetate.

\subsection{Statistical analysis}

Mean and mean standard errors were calculated for all variables in each of the experimental groups $(n=10)$. Twoway ANOVA followed by the Bonferroni post-hoc test was used to compare differences between groups in distinct conditions. Statistical Package for the Social Sciences (SPSS Inc., version 12.0) was used for all analysis. The significance level was set at $5 \%$.

\section{Results}

Body weights, absolute and relative heart weights and soleus muscle citrate synthase activity are shown in Table 2. In accordance with the well-described body mass and cardiac adaptations induced by endurance training, the 14 weeks of endurance running training decreased rat weight, and induced an increased in heart weight and heart/body ratio $(p<0.05)$. There was an increase in skeletal muscle citrate synthase activity in trained rats. This improved enzymatic activity in soleus reflects that endurance training

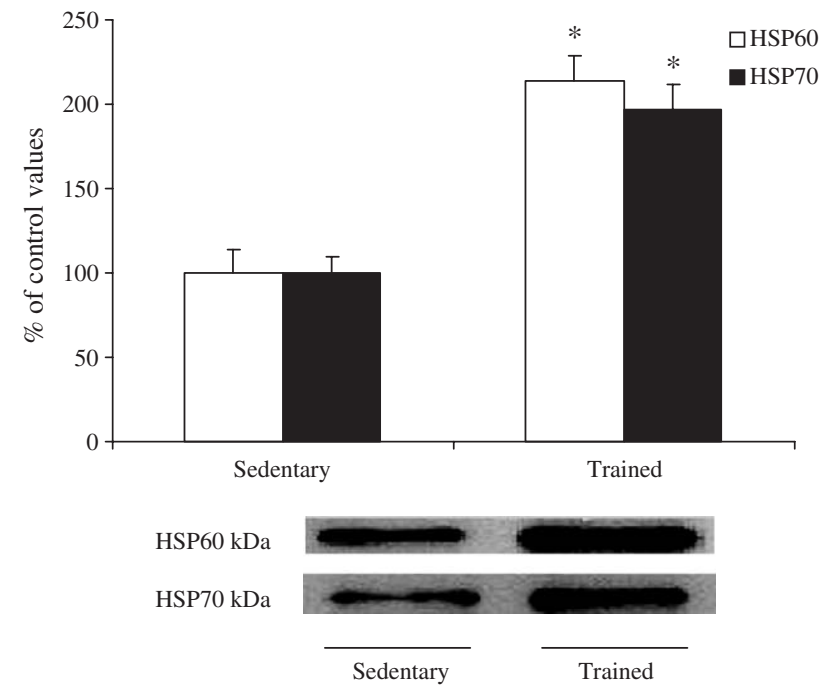

Fig. 3. Effect of endurance running training on the expression of heart mitochondria HSP60 and tissue homogenate HSP70. Immediately below the histogram, the panel shows a representative western blotting of HSP60 and HSP70 for each group as described in Methods. Values (mean and SEM) are expressed as percentage of control $* p<0.05$ trained vs. sedentary.

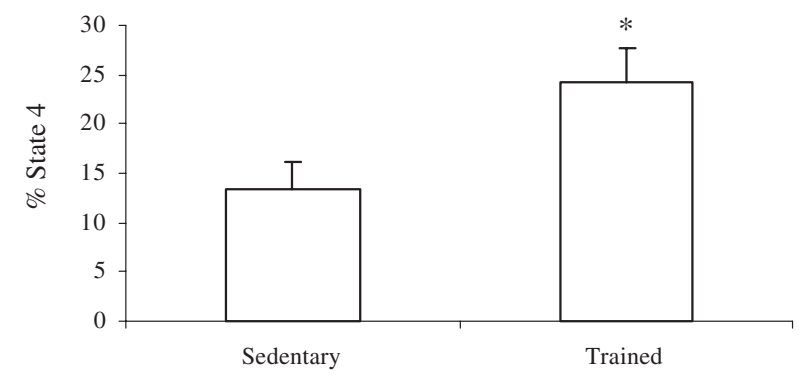

Fig. 4. Effect of endurance training on the inhibition of state 4 respiration with the addition of GDP $(1 \mathrm{mM})$. Magnitude of respiratory inhibition induced by GDP on state 4 respiration rate, which was expressed as the percentage of inhibition of the rate in state IV. ${ }^{*} p<0.05$ compared with sedentary group.

was an efficient chronic stimulus to ameliorate muscle oxidative metabolism.

As illustrated in Fig. 3, endurance running training elevated significantly the expression of basal levels of both heart mitochondria HSP60 and whole cardiac muscle homogenate HSP70. In fact, when compared to sedentary controls, increases of 114\% (HSP60) and 97\% (HSP70) were verified in trained groups.

The inhibition of state 4 respiration by $1 \mathrm{mM}$ GDP, a specific inhibitor of UCP, was significantly higher in heart mitochondria from trained animals than in heart mitochondria from sedentary animals. As illustrated in Fig. 4,

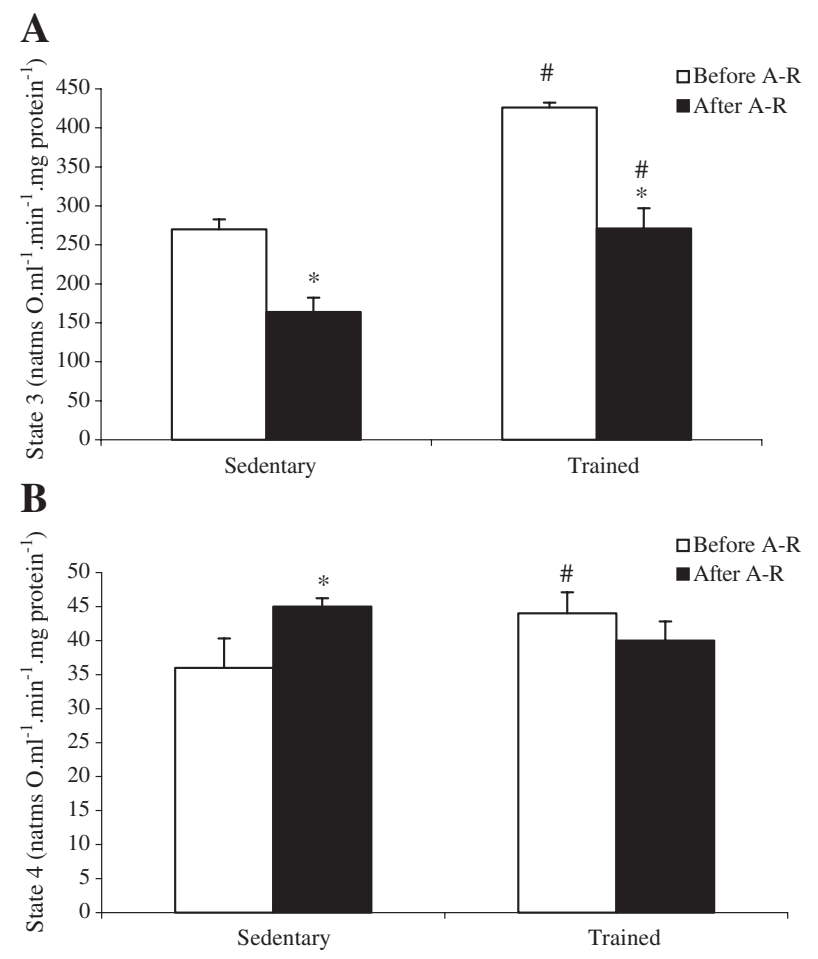

Fig. 5. Influence of A-R on the respiratory parameters of rat heart mitochondria respiring with glutamate $(10 \mathrm{mM})$ and malate $(5 \mathrm{mM})$ from both sedentary and trained hypertrophied rat hearts. (A) Respiratory rates in state 3. (B) Respiratory rates in state $4 .{ }^{*} p<0.05$ compared with respiratory rates before A-R; ${ }^{\#} p<0.05$ compared with respiratory rates of sedentary group. 
A

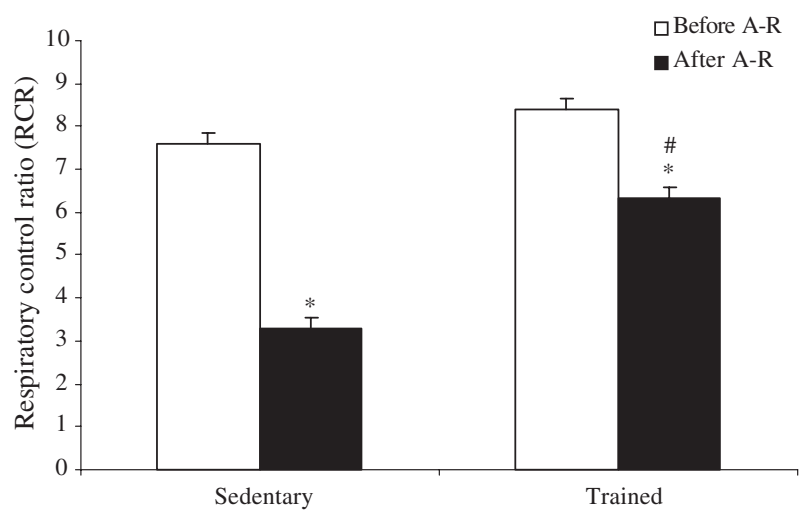

B

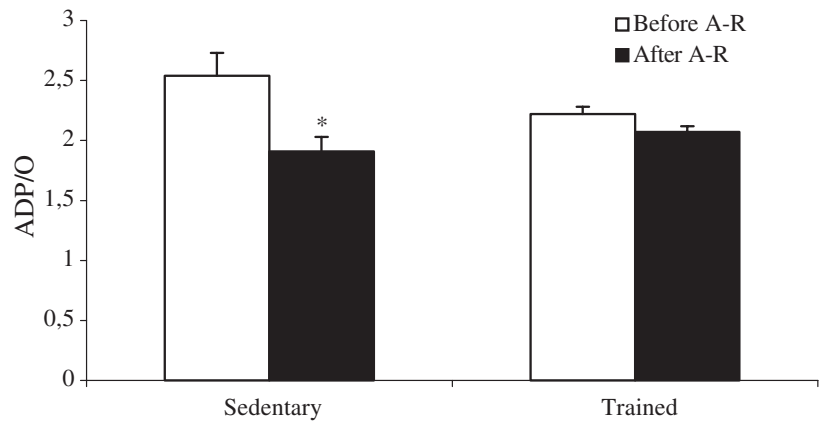

Fig. 6. Influence of A-R on RCR (A) and on ADP/O (B) of rat heart mitochondria incubated with the complex I-linked substrates glutamate (10 $\mathrm{mM})$ and malate $(5 \mathrm{mM})$ from both sedentary and trained hypertrophied rat hearts. ${ }^{*} p<0.05$ compared with respiratory rates before A-R; ${ }^{\#} p<0.05$ compared with respiratory rates of sedentary group.

respiratory rates in state IV upon addition of GDP were inhibited at percentages of $13.44 \%$ and $24.33 \%$ in sedentary and trained groups, respectively.

Mitochondrial respiratory parameters after the "timecontrol trial" were not significantly different from those obtained before anoxia. Actually, RCR and ADP/O calculated after A-R were not different from those obtained before A-R in both sedentary and trained groups.

Concerning the anoxia-reoxygenation data, the exposure of mitochondria to this insult induced a significant decrease in state 3 respiration rate in sedentary group (from $270.0 \pm 0.5$ to $164.0 \pm 18.3$ natms O. $\mathrm{ml}^{-1} \cdot \mathrm{min}^{-1} . \mathrm{mg}$ protein $^{-1}$ ) as well as in trained group (from $426.0 \pm 6.4$ to $271.0 \pm 2.6$ natms O. $\left.\mathrm{ml}^{-1} \cdot \mathrm{min}^{-1} . \mathrm{mg} \mathrm{protein}^{-1}\right)$. This can be a sign of respiratory chain dysfunction through intrinsic damage of its oxido-reductases and/or a limitation in the supply of reducing equivalents or even interpreted as an impairment of mitochondrial phosphorylation system. However, it is noteworthy the significant difference between heart mitochondria absolute state 3 respiratory rates from trained animals when compared to their sedentary counterparts, both before and after A-R (Fig. 5A). State 4 respiration was only increased after A-R in non-training group (from $36.3 \pm 4.3$ to $45.0 \pm 1.24$ natms O. $\mathrm{ml}^{-1} . \mathrm{min}^{-1} . \mathrm{mg} \mathrm{protein}^{-1}$ ), which means an increased permeability to protons through the mitochondrial inner membrane, whereas it further decreased slightly in mitochondria isolated from trained rats (from

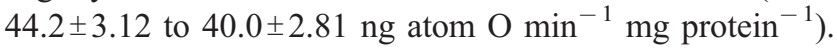
Endurance training remarkably increased the rate of oxygen consumption in state 4 respiration before A-R (Fig. 5B).

The absolute RCR values were significantly depressed by A-R, both in trained (from $8.38 \pm 0.46$ to $6.65 \pm 0.25$ ) and in sedentary groups (from $7.59 \pm 0.5$ to $3.31 \pm 0.24$ ) (Fig. $6 \mathrm{~A})$. Nevertheless, a noticeable decreased fall was verified after A-R in trained when compared to sedentary group when expressed as the ratio of $\mathrm{RCR}$ after/before A-R $(0.75 \pm 0.09$ vs. $0.43 \pm 0.07, p<0.05)$. Concerning $\mathrm{ADP} / \mathrm{O}$ ratio, and as can be seen from Fig. 6B, A-R induced significant decrease only in non-training group (from $2.54 \pm 0.19$ to $1.91 \pm 0.57)$. Endurance running training limited the fall in ADP/O induced by A-R (from $2.2 \pm 0.06$ to $2.07 \pm 0.05$ ). Accordingly, the ratio of $\mathrm{ADP} / \mathrm{O}$ after/before A-R was also closer to 1.0 in trained than in sedentary group $(0.93 \pm 0.08$ vs. $0.75 \pm 0.03)$.
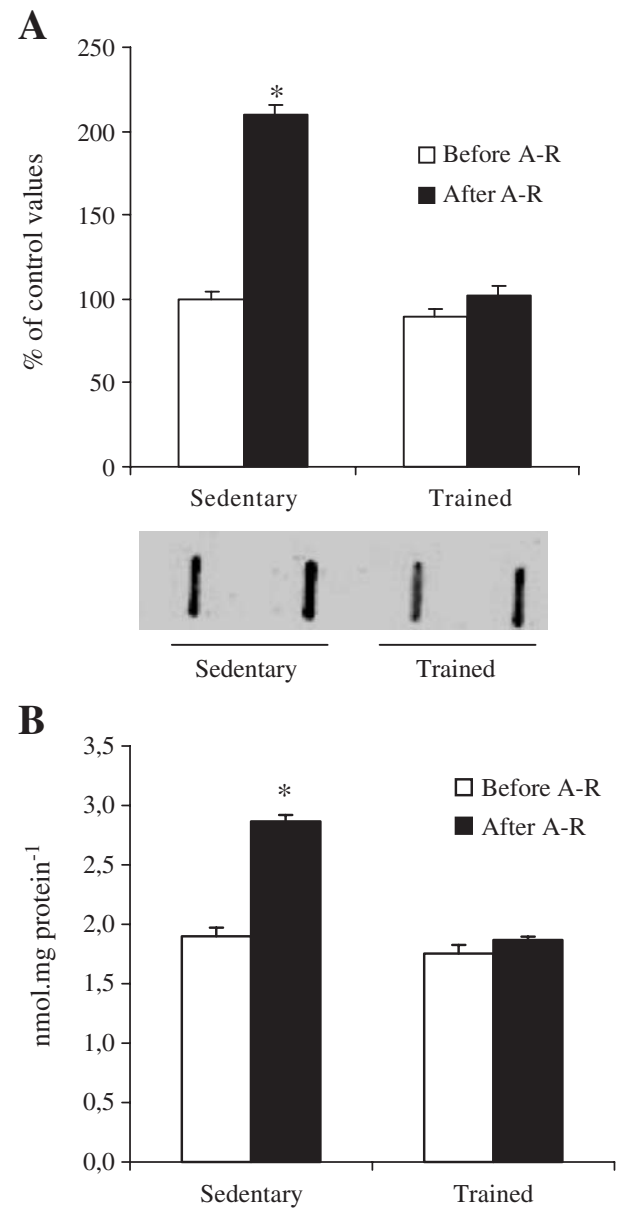

Fig. 7. Effect of training and A-R on the content of carbonyl derivatives (A) and malondialdehyde (B) of heart mitochondria. Immediately below the histogram A, the protein carbonyl formation panel shows a representative pattern of anti-denitrophenyl (DNP)-specific interaction with DNP for each group as described in Methods. Values (mean and SEM) are expressed as percentage of control. Sedentary (S), Trained (T). ${ }^{*} p<0.05$ before vs. after A-R. 
Concerning oxidative damage markers assayed both before and after A-R insult, namely protein carbonyl groups and MDA from rat heart mitochondria extracts, significant increases were observed in sedentary, whereas no significant changes were noted in trained group (Fig. 7).

\section{Discussion}

The use of A-R model of in vitro stimulation allows understanding the contribution of mitochondria to the trained hearts' tolerance against adverse stimuli. In fact, in studies that analyse the overall cardiac tissue response to deleterious stimuli, the complexity of the integrated cell defence systems limits the understanding and the precise description of the cell response mechanisms. Most of the times, differences in mitochondrial behaviour can only be uncovered after exposing the isolated mitochondrial fraction to stress conditions. One of the ways is by adding ADP, which mimics cellular need for extra energy production. Anoxia-reoxygenation of the isolated mitochondrial fraction is another inducer of stress that can be used to detect biochemical and functional changes present in mitochondrial fraction from different treated groups. In the present work, we proposed to study the protective role of endurance training on AR-induced mitochondrial dysfunction, a subjected only lightly explored in the literature. Our results showed that the A-R model is suitable to uncover differences between treatment groups.

As described in methods, we have verified in the "timecontrol trial" that incubation of mitochondria in the respiratory medium for $15-20 \mathrm{~min}$ did not alter respiratory features. This clear shows that aging of the mitochondrial fraction present in the reaction chamber was no cause of the differences observed upon A-R (Fig. 2). These data were in accordance with other reports in lung [29], brain [30] and liver $[14,15]$ isolated mitochondria, where there was no significant in vitro aging during respiratory assays.

To the best of our knowledge, this is the first study that analyses the effect of moderate endurance treadmill training on rat heart mitochondrial respiratory function. In accordance with A-R experiments in mitochondria derived from other tissues like brain and liver [14,15,30], although in contrast to other similar experiments using isolated lung mitochondria [29], the present study revealed that A-R impaired all the measured respiratory parameters in sedentary heart mitochondria, namely state 3 , state 4 , RCR and ADP/O ratios (Figs. 5 and 6 ). The state 3 decrease is usually interpreted as a sign of respiratory chain dysfunction through intrinsic damage of its oxido-reductases and/or a limitation of reducing equivalents [29,31] with consequent decline of supplied electrons to mitochondria electron transport chain (ETC), or to specific damage to the mitochondrial phosphorylation system (ATP synthase, phosphate transporters or the adenine nucleotide translocator). Nevertheless, despite the fact that the inhibition of state 3 by A-R occurred in both sedentary and trained mitochondria, state 3 was higher in trained than in sedentary group before A-R. Since we provided the same concentrations of glutamate-malate to mitochondria, one can argue that the higher phosphorylation rates in mitochondria from trained animals can be explained, at least partially, by the enhanced activities or differential expression of substrate translocators, by components of the ETC or by other mitochondrial metabolic pathways. As seen by the enhanced basal state 4 respiratory rates, heart mitochondria inner membrane damage induced by A- $\mathrm{R}$ in sedentary group resulted in an increased permeability to protons most likely due to oxidative stress-induced damage to membrane lipids or proteins [32] or by causing proton slippage through the ATP synthase or other proteins of the membrane respiratory complex $[29,31] . \mathrm{H}^{+}$leak (membrane damage) and/or $\mathrm{H}^{+}$ slip (modification of intrinsic $\mathrm{H}^{+}$stoichiometry of respiratory chain redox pumps and/or ATP synthase) led to a decrease in $\mathrm{ADP} / \mathrm{O}$ ratio, i.e., compromised the efficiency of oxidative phosphorylation. However, our data importantly demonstrated that endurance training minimized the increased rate in state 4 as well as the decreased state 3, $\mathrm{RCR}$ and $\mathrm{ADP} / \mathrm{O}$ caused by A-R, protecting rat heart mitochondria. This suggests an augmented resistance of the respiratory function in trained group against the referred stress stimuli.

Central to the considerations of this discussion is the property of mitochondria to be generators of oxygenderived free radicals, such as superoxide anion, during A$\mathrm{R}[14,15]$. The suggestion of an injury mechanism linked to oxidative protein modification and the protective effect of training are strongly supported by the observed significant increased formation of protein carbonyl derivatives in heart mitochondria from sedentary animals after A-R when compared with heart mitochondria from trained animals (Fig. 7). Moreover, there was a remarkable increase in the level of MDA produced during A-R in the sedentary group (Fig. 7), which was previously reported by others [33]. However, the present results showed that, with a similar tendency of protein damage, the levels of lipid peroxidation observed after A-R in the sedentary group were also significantly attenuated in heart mitochondria from the trained group. In fact, as polyunsaturated fatty acids are considered highly susceptible to ROS attack, the increased oxidative stress caused by A-R in sedentary group seem to lead to peroxidative modification of membrane lipids, as assessed by MDA formation, altering normal mitochondria respiratory function [34].

Cardiac mitochondria ETC produce ROS during basal state 4 , when the transmembrane electric potential $(\Delta \psi)$ is elevated above a determined critical threshold value. In fact, Korshunov et al. [35] demonstrated that small decreases in $\Delta \psi$ resulted in a strong inhibition of $\mathrm{H}_{2} \mathrm{O}_{2}$ formation in a mechanisms named by the authors as mild uncoupling. This mechanism would keep the $\Delta \psi$ below the critical threshold for augmented ROS production. In 
our study, as can be depicted from Fig. 4, the inhibition of state 4 respiration by GDP was significantly higher in heart mitochondria from trained animals when compared with heart mitochondria from sedentary prior to A-R. Despite the observation of Boss et al. [36] of a decrease in heart UCP2 mRNA after 8-week endurance training, which does not favour our findings, a higher GDP-mediated decrease in state 4 in trained group compared with sedentary was observed. This suggests that possibly training induced a mild decrease of $\Delta \psi$ through an UCP-related mechanism, regulating the electron flux through ETC and reducing the amount of free radicals produced. The protective outcome of this uncoupling-mediated effect was previously demonstrated by the addition of small concentrations of fatty acids with concomitant decrease in ROS formation and by the increased stimulation of $\mathrm{H}_{2} \mathrm{O}_{2}$ production in the presence of GDP in mitochondria containing UCP [37-39].

Among other possible cell and mitochondria defence systems, the up-regulation of mitochondria HSP60 and cardiac HSP70 mediated by endurance training that was found in our study (Fig. 3) could be also involved in mitochondrial protection against oxidative-based damage, contributing to minimize the decrease in the respiratory function of this organelle when submitted to A-R. In fact, it has been shown that HSP plays an essential role in keeping newly synthesized mitochondrial proteins (precursor proteins) in their correct unfolding conformation within the cytosol and transporting them into mitochondria [40]. Therefore, one could suggest that cytoplasmic HSP70 overexpression could enhance translocation of precursor ETC and other proteins into mitochondria, which could contribute to the improved respiratory function of trained organelles after A-R when compared to sedentary. Moreover, given that the levels of mitochondrial HSP60 increased in trained groups as well, it is possible that the improved assisted folding of proteins within mitochondria also afforded additional protection to mitochondria respiration of trained group. This relationship is consistent with the data from other studies in which mitochondria abnormal respiration induced by I-R was reduced in myocardium expressing high levels of HSP 70, 60 and 32 [41]. In the present study, the relationship between HSP expression and mitochondrial respiratory function provides some evidence that the up-regulation of the referred molecular chaperones may be involved in the preservation of mitochondria complexes' integrity and activity.

Another possible explanation for the higher respiratory function of trained mitochondria than sedentary controls, when submitted to A-R deleterious effects, could be associated with the training-mediated effect on the upregulation of mitochondrial antioxidant enzymes. Accordingly, this could have also been linked to HSP overexpression in trained group [42]. As previously reported by others $[16,43]$, endurance training ameliorated mitochondria antioxidant enzyme activity. This adaptation might also contribute to improve the capacity of mitochondria to counteract A-R-induced free radical-mediated malfunction. Similar findings were successfully obtained when isolated liver mitochondria demonstrated beneficial effects on the impaired respiratory rates caused by in vitro hypoxiareoxygenation in the presence of soluble antioxidants that were added during the incubation period [33].

In summary and regardless the hypothetical mechanisms, the results of the present study show that moderate endurance training improved heart mitochondrial respiratory function and increased their tolerance against A-R-induced dysfunction. Moreover, these findings suggest that mitochondrial adaptations might be involved in the welldescribed training-induced protection against cardiac deleterious insults.

\section{Acknowledgments}

We thankfully acknowledge the collaboration of Mrs. Celeste Resende and Mr. Serafim Pereira for their technical assistance regarding animals' care and training protocol.

\section{References}

[1] Dhalla NS, Elmoselhi AB, Hata T, Makino N. Status of myocardial antioxidants in ischemia-reperfusion injury. Cardiovasc Res 2000; $3: 446-56$.

[2] Jassem W, Fuggle SV, Rela M, Koo DD, Heaton ND. The role of mitochondria in ischemia/reperfusion injury. Transplantation 2002; 4:493-9.

[3] Ferrari R. The role of mitochondria in ischemic heart disease. J Cardiovasc Pharmacol 1996;S1-10.

[4] Cadenas E. Mitochondrial free radical production and cell signaling. Mol Aspects Med 2004;1-2:17-26.

[5] Wallace KB, Eells JT, Madeira VM, Cortopassi G, Jones DP. Mitochondria-mediated cell injury. Symposium overview. Fundam Appl Toxicol 1997;1:23-37.

[6] Powers SK, Lennon SL, Quindry J, Mehta JL. Exercise and cardioprotection. Curr Opin Cardiol 2002;5:495-502.

[7] Starnes JW, Bowles DK. Role of exercise in the cause and prevention of cardiac dysfunction. Exerc Sport Sci Rev 1995;349-73.

[8] Ji LL. Exercise-induced modulation of antioxidant defense. Ann NY Acad Sci 2002;82-92.

[9] Frenzel H, Schwartzkopff B, Holtermann W, Schnurch HG, Novi A, Hort W. Regression of cardiac hypertrophy: morphometric and biochemical studies in rat heart after swimming training. J Mol Cell Cardiol 1988;8:737-51.

[10] Powers SK, Demirel HA, Vincent HK, Coombes JS, Naito H, Hamilton KL, et al. Exercise training improves myocardial tolerance to in vivo ischemia-reperfusion in the rat. Am J Physiol 1998;5(Pt 2): R1468-77.

[11] Tonkonogi M, Sahlin K. Physical exercise and mitochondrial function in human skeletal muscle. Exerc Sport Sci Rev 2002;3:129-37.

[12] Leichtweis SB, Leeuwenburgh C, Parmelee DJ, Fiebig R, Ji LL. Rigorous swim training impairs mitochondrial function in postischaemic rat heart. Acta Physiol Scand 1997;2:139-48.

[13] Venditti P, Di Meo S. Antioxidants, tissue damage, and endurance in trained and untrained young male rats. Arch Biochem Biophys 1996; $1: 63-8$. 
[14] Du G, Mouithys-Mickalad A, Sluse FE. Generation of superoxide anion by mitochondria and impairment of their functions during anoxia and reoxygenation in vitro. Free Radic Biol Med 1998; 9:1066-74.

[15] Du G, Willet K, Mouithys-Mickalad A, Sluse-Goffart CM, DroyLefaix MT, Sluse FE. EGb 761 protects liver mitochondria against injury induced by in vitro anoxia/reoxygenation. Free Radic Biol Med 1999;5-6:596-604.

[16] Somani SM, Frank S, Rybak LP. Responses of antioxidant system to acute and trained exercise in rat heart subcellular fractions. Pharmacol Biochem Behav 1995;4:627-34.

[17] Samelman TR. Heat shock protein expression is increased in cardiac and skeletal muscles of Fischer 344 rats after endurance training. Exp Physiol 2000;1:97-102.

[18] Sammut IA, Harrison JC. Cardiac mitochondrial complex activity is enhanced by heat shock proteins. Clin Exp Pharmacol Physiol 2003; $1-2: 110-5$.

[19] Camper-Kirby D, Welch S, Walker A, Shiraishi I, Setchell KD, Schaefer E, et al. Myocardial Akt activation and gender: increased nuclear activity in females versus males. Circ Res 2001;10:1020-7.

[20] Lowry OH, Rosenbrough N, Farr AL, Radall RJ. Protein measurement with the folin phenol reagent. J Biol Chem 1951;193:265-75.

[21] Bhattacharya SK, Thakar JH, Johnson PL, Shanklin DR. Isolation of skeletal muscle mitochondria from hamsters using an ionic medium containing ethylenediaminetetraacetic acid and nagarse. Anal Biochem 1991;2:344-9.

[22] Estabrook R. Mitochondrial respiratory control and the polarographic measurement of ADP/O ratios. Methods Enzymol 1967;41-7.

[23] Skulachev VP. Membrane-linked systems preventing superoxide formation. Biosci Rep 1997;3:347-66.

[24] Rohn TT, Hinds TR, Vincenzi FF. Ion transport ATPases as targets for free radical damage. Protection by an aminosteroid of the $\mathrm{Ca}^{2+}$ pump ATPase and $\mathrm{Na}^{+} / \mathrm{K}^{+}$pump ATPase of human red blood cell membranes. Biochem Pharmacol 1993;3:525-34.

[25] Buege JA, Aust SD. Microsomal lipid peroxidation. Methods Enzymol 1978;302-10.

[26] Laemmli UK. Cleavage of structural proteins during the assembly of the head of bacteriophage T4. Nature 1970;259:680-5.

[27] Locke M, Noble EG, Atkinson BG. Exercising mammals synthesize stress proteins. Am J Physiol 1990;4(Pt 1):C723.

[28] Coore HG, Denton RM, Martin BR, Randle PJ. Regulation of adipose tissue pyruvate dehydrogenase by insulin and other hormones. Biochem J 1971;1:115-27.

[29] Willet K, Detry O, Sluse FE. Resistance of isolated pulmonary mitochondria during in vitro anoxia/reoxygenation. Biochim Biophys Acta $2000 ; 2-3: 346-52$.

[30] Morin C, Zini R, Simon N, Tillement JP. Dehydroepiandrosterone and alpha-estradiol limit the functional alterations of rat brain mitochon- dria submitted to different experimental stresses. Neuroscience 2002; 2:415-24.

[31] Willet K, Detry O, Lambermont B, Meurisse M, Defraigne JO, SluseGoffart $\mathrm{C}$, et al. Effects of cold and warm ischemia on the mitochondrial oxidative phosphorylation of swine lung. Transplantation 2000;4:582-8.

[32] Choksi KB, Boylston WH, Rabek JP, Widger WR, Papaconstantinou J. Oxidatively damaged proteins of heart mitochondrial electron transport complexes. Biochim Biophys Acta 2004;2:95-101.

[33] Schild L, Reinheckel T, Wiswedel I, Augustin W. Short-term impairment of energy production in isolated rat liver mitochondria by hypoxia/reoxygenation: involvement of oxidative protein modification. Biochem J 1997;205-10.

[34] Halliwell B, Gutteridge JM. Free radicals in biology and medicine. Oxford: Clarendon Press; 1999.

[35] Korshunov SS, Skulachev VP, Starkov AA. High protonic potential actuates a mechanism of production of reactive oxygen species in mitochondria. FEBS Lett 1997;1:15-8.

[36] Boss O, Samec S, Desplanches D, Mayet MH, Seydoux J, Muzzin P, et al. Effect of endurance training on mRNA expression of uncoupling proteins 1, 2 and 3 in the rat. FASEB J 1998;3:335-9.

[37] Korshunov SS, Korkina OV, Ruuge EK, Skulachev VP, Starkov AA. Fatty acids as natural uncouplers preventing generation of $\mathrm{O}_{2}^{-}$and $\mathrm{H}_{2} \mathrm{O}_{2}$ by mitochondria in the resting state. FEBS Lett 1998;2-3: $215-8$.

[38] Kowaltowski AJ, Costa AD, Vercesi AE. Activation of the potato plant uncoupling mitochondrial protein inhibits reactive oxygen species generation by the respiratory chain. FEBS Lett 1998;2:213-6.

[39] Negre-Salvayre A, Hirtz C, Carrera G, Cazenave R, Troly M, Salvayre $\mathrm{R}$, et al. A role for uncoupling protein-2 as a regulator of mitochondrial hydrogen peroxide generation. FASEB J 1997;10: $809-15$.

[40] Deshaies RJ, Koch BD, Werner-Washburne M, Craig EA, Schekman R. A subfamily of stress proteins facilitates translocation of secretory and mitochondrial precursor polypeptides. Nature 1988;6167:800-5.

[41] Sammut IA, Jayakumar J, Latif N, Rothery S, Severs NJ, Smolenski RT, et al. Heat stress contributes to the enhancement of cardiac mitochondrial complex activity. Am J Pathol 2001;5:1821-31.

[42] Suzuki K, Murtuza B, Sammut IA, Latif N, Jayakumar J, Smolenski RT, et al. Heat shock protein 72 enhances manganese superoxide dismutase activity during myocardial ischemia-reperfusion injury, associated with mitochondrial protection and apoptosis reduction. Circulation 2002;12(Suppl. 1):I270.

[43] Moran M, Delgado J, Gonzalez B, Manso R, Megias A. Responses of rat myocardial antioxidant defences and heat shock protein HSP72 induced by $12-$ and $24-w e e k$ treadmill training. Acta Physiol Scand 2004;2:157-66. 